\title{
Freiheit, Institutionen, abstrakte Gefährdungsdelikte: Ein neuer Prototyp des Wirtschaftsstrafrechts?
}

\section{Aktueller Anlass und grundsätzliche Fragen}

Abstrakte Gefährdungsdelikte sind seit langem Bestandteil des Strafrechts, zumal des Wirtschaftsstrafrechts. So macht sich wegen eines Kapitalanlagebetruges (§ 264a StGB) derjenige strafbar, der in einem Verkaufsprospekt falsche Angaben zu Wertpapieren macht. Dass die Wertpapiere tatsächlich gekauft werden und dies $\mathrm{zu}$ einem $\mathrm{zu}$ hohen Preis, ist nicht erforderlich. ${ }^{1}$ Das Verbot bezieht sich auf die (gefährliche) Handlung, ab deren Vornahme der Handelnde das Geschehen aus der Hand gibt, so dass es nun nicht mehr an ihm liegt, ob eine Person konkret gefährdet oder geschädigt wird, ob also jemand ein Wertpapier (überteuert) erwirbt. Das alles ist nicht neu und doch ist das von den Organisatoren der Tagung definierte Thema „Abstrakte Gefährdungsdelikte - der zunehmende Prototyp des Wirtschaftsstrafrechts?“ von aktueller Bedeutung. Denn es enthält sowohl eine aktuelle als auch eine nach wie vor klärungsbedürftige Komponente. Erstens ist zu fragen, ob tatsächlich ein neuer und nachhaltiger Trend zur Schaffung abstrakter Gefährdungsdelikte im Wirtschaftsstrafrecht zu erwarten ist. Zweitens lässt sich das Fragezeichen hinter dem Vortragstitel auf die Legitimität einer solchen Entwicklung beziehen und also fragen, ob die Schaffung weiterer abstrakter Gefährdungsdelikte den - schon vor längerem von der sog. „Frankfurter Schule“2 konstatierten - „unmöglichen Zustand des Strafrechts“"3 verstärkt oder ob sich für die Implementierung weiterer abstrakter Gefährdungsdelikte nicht doch gute, strafrechtstheoretisch werthaltige Gründe finden lassen.

Beiden Fragen soll im Folgenden nachgegangen werden. Die Untersuchung gelangt zum einen zu der Prognose, dass mittelfristig von einer Zunahme abs-

1 Statt vieler Wittig Wirtschaftsstrafrecht, 2. Aufl. 2011, § 6 Rn. 8. S. aber auch Rotsch in: Momsen/Grützner, Wirtschaftsstrafrecht, 2013, S. 13, 21.

2 Dazu umfassend Jahn/Ziemann in: Fachbereich Rechtswissenschaft der Goethe Universität Frankfurt a.M. (Hrsg.), 100 Jahre Rechtwissenschaft in Frankfurt, 2014, S. $299 \mathrm{ff}$.

3 S. den gleichnamigen Titel sowie das Vorwort des vom Institut für Kriminalwissenschaft herausgegebenen Bandes, Vom unmöglichen Zustand des Strafrechts, 1995, S. 5 f. 
trakter Gefährdungsdelikte auszugehen ist. Zum anderen wird die - wirtschaftspolitisch gesprochen - ordoliberale These aufgestellt und begründet, dass eine weitere Implementierung abstrakter Gefährdungsdelikte zum Schutz freiheitserhaltender und -ermöglichender Institutionen notwendig und (verfassungsrechtlich sowie strafrechtstheoretisch) zulässig sein kann.

\section{Abwendung vom Erfolgsdelikt und Hinwendung zum abstrakten Gefährdungsdelikt}

\section{Stringente Evolution des Wirtschaftsstrafrechts?}

Wenden wir uns der ersten Frage zu: Ist zu erwarten, dass das abstrakte Gefährdungsdelikt zum neuen Leitdeliktstyp des Wirtschaftsstrafrechts avanciert? Glaubt man der Evolutionstheorie des Rechts in der Spielart ihres berühmtesten strafrechtlichen Vertreters, Franz von Liszt, lässt sich die Antwort auf diese Frage am geltenden Recht und seiner Genese ablesen. Wegen der „kausalen Gesetzmäßigkeit allen Geschehens“ könne, so Liszt, vom gewordenen und seienden Recht auf das werdende Recht geschlossen werden. ${ }^{4}$ Träfe dies zu, dann ließe sich auch aus der zunehmenden Implementierung von abstrakten Gefährdungsdelikten in der Vergangenheit auf eine künftige Entwicklung schließen, die auf das abstrakte Gefährdungsdelikt als neuen Prototyp des Wirtschaftsstrafrechts hinausliefe. Doch so einfach liegen die Dinge nicht, wie schon Liszt selbst erleben musste. ${ }^{5}$ Denn die Entwicklung von Recht und Gesellschaft ist stets komplexer als Entwicklungsnarrative glauben machen. Das hat seinen Grund darin, dass das Strafrecht als Teil der „Selbstbewegung der großen Ideen" ${ }^{* 6}$ den sozialen, kulturellen und politischen Paradigmen seiner Zeit folgt.

4 S. nur v. Liszt ZStW 26 (1906), 553, 556; ders. JW 1918, 754; ders. Vom Völkerbund zur Staatengemeinschaft, 1918, S. 5f. Zum Ganzen Herrmann Das Standardwerk. Franz von Liszt und das Völkerrecht, 2001, S. 185 ff., 190: „Er (der Entwicklungsgedanke, M.K.) stellt gleichsam das Leitmotiv in seinen wissenschaftlichen Bemühungen dar." Umfassend dazu demnächst Pawlik in: Koch/Löhnig (Hrsg.), Die v. Liszt-Schule und die Entstehung des modernen Strafrechts, 2015.

5 Zum Ausbleiben rechtspolitischer Erfolge Liszts statt vieler Herrmann NJW 2001, 2854, 2855. Am Beispiel des von Liszt propagierten einheitlichen mitteleuropäischen Strafrechts Kubiciel JZ 2015, $63 \mathrm{ff}$.

6 Braun Einführung in die Rechtsphilosophie, 2006, S. 393. Aus strafrechtswissenschaftlicher Sicht Hilgendorf JRE 2003, 83, 88. 
Daher verliert sich, so Max Weber, irgendwann der Weg, den diese Paradigmen weisen, in der Dämmerung: „Das Licht der großen Kulturprobleme ist weiter gezogen. Dann rüstet sich auch die Wissenschaft, ihren Standort und ihren Begriffsapparat zu wechseln (...). Sie zieht jenen Gestirnen nach, welche allein ihrer Arbeit Sinn und Richtung zu weisen vermögen. "7 Anders als die Planeten eines Sonnensystems, folgen die „Gestirne“ des Systems Strafrecht aber keiner vorgezeichneten Bahn. Vielmehr spiegelt das Strafrecht gesellschaftliche und politische Entwicklungen, die sich eben nicht gradlinig auf ein Ziel zubewegen, sondern tentativ verlaufen, gelegentlich in Extreme verfallen und an ihrem Scheitelpunkt wieder die gegenläufige Entwicklungslinie einschlagen. So wechseln sich in der Wirtschaftspolitik und dem - die Wirtschaftspolitik umsetzenden Wirtschaftsrecht - Phasen der Deregulierung mit Perioden der ReRegulierung ab. Auch das Wirtschaftsstrafecht folgt keiner stringenten kriminalpolitischen Linie, die auf eine immer dichtere Regulierung zuläuft. Beide Rechtsmaterien, das Wirtschaftsrecht und das Wirtschaftsstrafrecht, kennen vielmehr auch Regulierungspausen. Daher ist die Entwicklung des „Dings“ hier: des Wirtschaftsstrafrecht - „nichts weniger als sein progressus, sondern die Aufeinanderfolge von mehr oder minder tiefgehenden, mehr oder minder voneinander unabhängigen, an ihm sich abspielenden ÜberwältigungsProzessen (...)“. ${ }^{8}$ Die Entwicklung des Wirtschaftsstrafrechts ist, so lässt sich Nietzsche vor unserem Hintergrund verstehen, vor allem Folge eines Ringens um die wirtschafts- und kriminalpolitische Deutungsherrschaft. Schon dieser eminent politische Charakter des Vorgangs spricht gegen die Annahme, die Rechtsentwicklung folge einer inneren Teleologie. Mit Hilfe simplifizierender Evolutionstheorien lässt sich die Frage, ob sich das abstrakte Gefährdungsdelikt als Prototyp des Wirtschaftsstrafrechts durchsetzen wird, folglich nicht beantworten.

Es kommt hinzu, dass das (Straf-)Recht Veränderungen in seiner Systemumwelt, namentlich der Wirtschaftspolitik, nicht einfach übernimmt, sondern diesen gewissermaßen mit Brechungen folgt. Änderungen in der Umwelt nimmt das System „Strafrecht“ zwar zur Kenntnis, indem neue außerstrafrechtliche Bezugsnormen in die Auslegung von Straftatbeständen einbezogen oder zusätz-

7 Vgl. Weber Die ,Objektivität‘ sozialwissenschaftlicher und sozialpolitischer Erkenntnis, in: Winckelmann (Hrsg.), Max Weber, Gesammelte Aufsätze zur Wissenschaftslehre, 1982, S. 146, S. 214.

8 Nietzsche Zur Genealogie der Moral, in: Vormbaum (Hrsg.), Strafrechtsdenker der Neuzeit, 1998, S. 500, 507. Zu Nietzsche Bung ZStW 119 (2007), 120 ff.; Müller/Dietz GA 2015, 160 ff. 
liche Straftatbestände geschaffen werden. Doch folgt das Strafrecht bei der Rezeption dieser Änderungen seiner eigenen Verarbeitungslogik. ${ }^{9}$ Prägend für die spezifische Systemlogik des Strafrechts ist der rechtskulturelle Überlieferungszusammenhang, in dem sich das Verständnis vom Strafrecht und das Selbstverständnis der Strafrechtswissenschaft herausgebildet haben. ${ }^{10}$ Kernbestandteil auch des heutigen Strafrechtsverständnis ist das Tat- und Schuldprinzip. Dieses Prinzip sowie die daran anknüpfende Warnung vor der Vorverlagerung der Strafbarkeit haben im kriminalpolitischen Diskurs eine Eigenschwere, die eine Entwicklung hin zu abstrakten Gefährdungsdelikten abbremsen und die Entwicklungsrichtung verändern kann. Mehr noch: Diese Topoi taugen ohne Weiteres dazu, die Umkehr der rechtspolitischen Entwicklung zu begründen, sollte die Gesellschaft in eine Phase der Deregulierung oder in eine Regulierungspause eintreten und das abstrakte Gefährdungsdelikt seinen Reiz verlieren. Das Tat- und Schuldprinzip wäre dann zwar nicht der Grund für die Abkehr vom abstrakten Gefährdungsdelikt, gäbe aber eine gute Begründung für diesen Schritt ab.

\section{Indizien für den Ausbau des Systems abstrakter Gefährdungsdelikte}

Wie wir sehen, wird die Evolution des (Wirtschafts-)Strafrechts von so vielfältigen, teils gegenläufigen Faktoren beeinflusst, dass keine Rede von einem „nicht umkehrbaren Wandel des Rechts (...) mit bestimmter Richtung“'11 - hier: weg vom Erfolgsdelikt und hin zum abstrakten Gefährdungsdelikt - sein kann. Gleichwohl gibt es nicht wenige Indizien, die einen - zumindest mittelfristig anhaltenden - Trend zum Ausbau des Systems abstrakter Gefährdungsdelikte im Wirtschaftsstrafrecht nahelegen. ${ }^{12}$

9 Dazu Kölbel ,Cultural Lag‘ und Normevolution. Systemtheoretische Überlegungen am Beispiel des Wirtschaftsstrafrechts, in: Kodalle/Rosa (Hrsg.), Rasender Stillstand. Beschleunigung des Wirklichkeitswandels, 2008, S. 69, 72, 73ff.; Vesting Rechtstheorie, 2007, S. $137 \mathrm{ff}$.

10 S. dazu Fikentscher Wissenschaft und Recht, in: Engel/Schön (Hrsg.), Das Proprium der Rechtswissenschaft, 2007, S. 77, 79. Zur Traditionsbindung der Rechtswissenschaft auch Dedek Rechtswissenschaft 1 (2010), S. 58. Zum Begriff des rechtswissenschaftlichen Überlieferungszusammenhangs Larenz Methodenlehre der Rechtswissenschaft, 5. Aufl. 1983, S. 209.

11 So die Rechtsevolution definierend Henke Über die Evolution des Rechts, 2010, S. 211.

12 Einen Trend zur Normierung abstrakter Gefährdungsdelikte konstatiert auch Rotsch (Fn. 1). 


\section{a) Beweisprobleme bei Erfolgsdelikten}

Verbreitet wird dieser Trend mit der Hypothese unterlegt, der Gesetzgeber wolle mit Hilfe der Gefährdungsdelikte jene Beweisprobleme umgehen, die Erfolgsdelikte aufwerfen. ${ }^{13}$ So ließe sich an ein Gefährdungsdelikt denken, das die Untreue funktional ersetzt, indem es Probleme bei der Bestimmung der Schadenshöhe oder der Zurechnung des Schadens zum pflichtwidrigen Verhalten eines konkreten Verursachers umgeht. Tatsächlich wechselt der Untreuetatbestand gegenwärtig sein Image: Stand er lange im Ruf eines zu beinahe jedem Fall der Wirtschaftskriminalität passenden Allzweckinstruments der Strafverfolgung, ${ }^{14}$ scheinen die vom BGH und BVerfG aufgestellten Hürden einen revisionsfesten Nachweis von Untreuevorsatz und Schaden in vielen Fällen nicht mehr zuzulassen. Dies gilt keineswegs nur für „Großschadenslagen“ wie jene der Finanzkrise; ${ }^{15}$ vielmehr scheinen auch alltägliche Pathologien des modernen Wirtschaftslebens nur noch mit Mühe in einer verfassungsgerichtsfesten Weise unter § 266 StGB subsumiert werden zu können. ${ }^{16}$ Versagt das Strafrecht aber nicht nur in einer Ausnahmesituation, ${ }^{17}$ sondern in der Normallage, weil sich über längere Zeiträume eine Vielzahl von Fällen, die von der Öffentlichkeit als strafwürdig erachtet werden, nicht mehr unter Tatbestände wie § 266 StGB fassen, ist der gesetzgeberische Schritt $\mathrm{zu}$ anspruchsärmeren Gefährdungsdelikten vorgezeichnet. Es überrascht daher nicht, dass die Justizministerkonferenz bereits die Idee ventiliert hat, schon die vorsätzliche Verletzung kaufmännischer Pflichten unabhängig vom Eintritt eines (Gefährdungs-)Schadens zu kriminalisieren. Indes steht der Vorschlag auf schwachen Füßen. Wenn das materielle Strafrecht geändert werden soll, um prozessuale Beweisprobleme zu lösen, ${ }^{18}$ setzt das, schon aus verfassungsrechtlichen Gründen, zweierlei voraus: valide Informa-

13 S. bereits Lüderssen in: Kempf/Lüderssen/Volk (Hrsg.), Die Handlungsfreiheit des Unternehmers, 2009, S. 241, 255f.; Prittwitz Strafrecht und Risiko, 1993, S. 249. Zusammenfassend Rotsch (Fn. 1), S. 22.

14 Vgl. Ransiek ZStW 116 (2004), 634: „passt immer“. S. ferner Bernsmann GA 2007, 219; ders. GA 2009, 296; Beulke FS Eisenberg, 2009, S. 245, 266.

15 Dazu etwa Jahn JZ 2011, 340 ff.; Kubiciel ZIS 2013, 53ff.; Lüderssen in: Kempf/Lüderssen/ Volk (Hrsg.), Die Finanzkrise, das Wirtschaftssystem und die Moral, 2010, S. 211 ff.; Rönnau in: Schünemann (Hrsg.), Die sogenannte Finanzkrise, Systemversagen oder global organisierte Kriminalität?, 2010, S. 43, 62; Schröder Handbuch Kapitalmarkstrafrecht, 3. Aufl. 2015, S. 357 ff., 376 ff. Allgemeiner Prittwitz (Fn. 13), S. 349, der betont, tradierte Zurechnungsstrukturen passten nicht zu globalen Zusammenhängen.

16 Vgl. Fischer StGB, 62. Aufl. 2015, § 266 Rn. 163f. m.w.N.

17 Zur Finanzkrise als Ausnahmezustand Kubiciel Nomos - Kansai Law Journal (2013), S. 21 ff.

18 Krit. Weigend Festschrift Triffterer 1996, S. $695 \mathrm{ff}$. 
tionen über die tatsächliche Existenz der behaupteten Probleme ${ }^{19}$ und zudem die Prüfung, ob sich den Problemen nicht durch weniger schwerwiegende Maßnahmen - Anpassung der Zurechnungsvoraussetzungen an die Besonderheiten des Wirtschaftsrechts - abhelfen lässt. Der Idee der Justizministerkonferenz, eine untreueanaloge Strafvorschrift mit abgesenkten Tatbestandsvoraussetzungen zu schaffen, sind bislang keine Taten gefolgt. Auch die Reaktionen des Strafgesetzgebers auf die Finanzkrise sind bislang ausgesprochen verhalten. ${ }^{20}$

\section{b) Der Einfluss des Europäischen Strafrechts}

Das Ziel, Beweisprobleme zu umgehen, ist jedoch nicht die einzige Triebfeder einer Entwicklung hin zum abstrakten Gefährdungsdelikt als neuem Prototyp des Wirtschaftsstrafrechts. Eine meines Erachtens wirkmächtigere, wenn auch weniger beachtete Quelle neuer abstrakter Gefährdungsdelikte ist das europäische Recht. Abstrakte Gefährdungsdelikte gelten auf internationaler und europäischer Ebene als angemessene Instrumente zum Schutz marktwirtschaftlicher Institutionen. ${ }^{21}$ Diese Überzeugung wirkt über internationale Konventionen und europäische Richtlinien auch auf das deutsche Recht ein. Tatsächlich haben schon heute nicht wenige Gefährdungsdelikte im Wirtschafts- und Umweltstrafrecht ein europarechtliches Fundament. ${ }^{22}$ Um ein aktuelles Beispiel aufzugreifen: Die Verschärfung des Tatbestandes der Bestechlichkeit und Bestechung im geschäftlichen Verkehr dient vor allem der Umsetzung europäischer Vorgaben ${ }^{23}$ und trägt der im EU Anti-Corruption Report geäußerten Kritik Rechnung. ${ }^{24}$

19 Zur Bedeutung der Wirtschaftswissenschaften für das Wirtschaftsstrafrecht vgl. Tiedemann Wirtschaftsstrafrecht - Allgemeiner Teil, 4. Aufl. 2014, S. 15.

20 Treffend Schröder WM 2014, 100, 101f.: Der Gesetzgeber habe - trotz der öffentlichen Stimmung - auf „hektische Reaktionen“ verzichtet. Mit dem Klischee eines dem „Volkszorn“ nacheilenden Gesetzgebers spielend hingegen Brand ZVglRWiss 113 (2014), 142, 143.

21 Tiedemann (Fn. 18), S. 80.

22 Umfassende Übersicht bei Dannecker/Bülte in: Wabnitz/Janovsky (Hrsg.), Handbuch des Wirtschafts- und Steuerstrafrechts, 4. Aufl. 2014, S. 79, $115 \mathrm{ff}$.

23 Rahmenbeschluss des Rates 2003/568/JI vom 22. Juli 2003 zur Bekämpfung der Bestechung im privaten Sektor, ABl. Nr. L 192, S. 54. Krit. dazu Gaede ZWiSt 2014, 280 ff., anderer Ansicht Kubiciel ZIS 2014, 667, 668f.

24 Annex Germany to the Report from the Commission to the Council and the European Parliament - EU Anti-Corruption Report, COM (2014) 38final, S. 6f.; zum Inhalt Kubiciel/Spörl Journal of Business Compliance 2014, 5. 


\section{c) Zunahme wirtschaftslenkender Primärnormen}

Der bedeutendste Impuls zur Schaffung neuer abstrakter Gefährdungsdelikte geht jedoch weder vom deutschen noch vom europäischen Strafgesetzgeber aus, sondern von der Schaffung immer neuer wirtschaftslenkender Primärnormen und der damit verbundenen Komplexitätssteigerung der Ordnungssysteme. Beispielhaft: Wenn die Bewirtschaftung natürlicher Ressourcen - Luft, Wasser, Boden - staatlich verwaltet wird und wenn bei Bewirtschaftungsentscheidungen ökologische, ökonomische und soziale Gesichtspunkte berücksichtigt werden dürfen, ist es auch strafrechtlich mit einfachen Erfolgsdelikten - Gewässerverunreinigung - nicht mehr getan. Vielmehr liegt es nahe, die teils in Verwaltungsakten enthaltenen einzelnen Primärnormen durch akzessorische Straftatbestände, Sekundärnormen, zu flankieren. Der Schutz konkreter Rechtsgüter tritt hinter den Normenschutz in diesen Fällen deutlich zurück. ${ }^{25}$ Ähnliches gilt für eine Vielzahl von Bereichen des Haupt- und Nebenstrafrechts, in denen der Strafgesetzgeber auf außerstrafrechtliche Primärnormen Bezug nimmt, um deren Geltung durch die Strafandrohung zu sichern. Der nächste Schritt dieser Entwicklung zeichnet sich an § 54a KWG ab. Das Kreditwesen und seine Regulierung haben offenbar bereits einen Grad der Komplexität erreicht, der eine Steuerung von Risiken allein mit Hilfe wirtschaftslenkender Primärnormen, an die eine strafrechtliche Sekundärnorm anknüpft, ausschließt. § 54a KWG flankiert daher - anders als etwa das Umweltstrafrecht - keine konkrete Primärnorm, sondern verlangt die Vorhaltung von Compliance-Standards, welche die Einhaltung der bankrechtlichen Primärnormen in Kreditinstituten gewährleisten sollen. ${ }^{26}$ Der Straftatbestand enthält, aus der Perspektive des Rechtsguts betrachtet, nur noch eine Tertiärnorm. Dies ist, wiederum vom Standpunkt der Rechtsgüterschutzlehre betrachtet, eine hochgradig bedenkliche Entwicklung. In wirtschaftspolitischer Hinsicht stellt der Tatbestand hingegen den Versuch dar, den Primat der Politik über die Finanzwirtschaft auch auf Tätigkeitsfeldern $\mathrm{zu}$ demonstrieren, ${ }^{27}$ in denen eine Risikosteuerung durch andere strafrechtliche Mittel nicht möglich erscheint.

25 Näher dazu Kubiciel Die Wissenschaft vom Besonderen Teil des Strafrechts, 2013, S. $262 \mathrm{ff}$. Zur h.M. Saliger Umweltstrafrecht, 2012, S. $18 \mathrm{ff}$.

$26 \mathrm{Vgl}$. Hamm in: Kempf/Lüderssen/Volk (Hrsg.), Unternehmenskultur und Wirtschaftsstrafrecht, 2015, S. 79, 82, der den „extremen“ Blankettcharakter des § 54a KWG und die Besonderheiten der Regelungsmaterie in Abgrenzung vom umweltverwaltungsakzessorischen Umweltstrafrecht betont (S. 90).

27 Vgl. Mansdörfer in: Kempf/Lüderssen/Volk (Fn. 26), 197, 205f. - Einen „traurigen Höhepunkt“ gesetzgeberischen „Versagens“ konstatiert hingegen Brand ZVglRWiss 113 (2014), 142, 145; ähnlich scharf Kasiske ZIS 2013, 257, 264. Krit. auch Hamm/Richter WM 2013, 865 ff.; Jahn 


\section{Der Einfluss der Strafrechtswissenschaft}

Diese Entwicklung hat die Strafrechtswissenschaft nicht aufhalten können; ihre lange herrschenden Konzeptionen - insbesondere die Rechtsgüterschutzlehre $^{28}$ - hatten diesem Trend auch wenig entgegenzusetzen. So kann eine präventionstheoretische Rechtfertigung der Strafe die Bedeutung des tatbestandlichen Erfolges nicht abbilden: Denn verbieten lässt sich nur die Vornahme einer gefährlichen Handlung, nicht der Eintritt eines Erfolges. Folgt man der Logik der Präventionstheorien - jedenfalls der negativen Generalprävention und der Spezialprävention - ist das Erfolgsdelikt der Problemfall, nicht das Gefährdungsdelikt. Lässt sich aber die Kritik der Strafrechtswissenschaft an der Gesetzgebung nicht bruchlos mit den von ihr verwendeten Grundlagenkonzepten (Rechtsgüterschutz, Prävention) vereinbaren, kann es nicht überraschen, wenn der Gesetzgeber seine kriminalpolitischen Entscheidungen unbeeinflusst von der wissenschaftlichen Kritik trifft. ${ }^{29}$

\section{Fazit}

Auf die Frage, ob das abstrakte Gefährdungsdelikt zum neuen Prototyp des Wirtschaftsstrafrechts avanciert, lässt sich - bei aller Vorsicht - folgende Antwort formulieren: Da sich den Steuerungsproblemen, welche die Wirtschaft aufwirft, nicht mit einfachen Erfolgsdelikten begegnen lässt und wir uns in einer Phase der Regulierung befinden, ist mit einer Zunahme von wirtschaftslenkenden Primärnormen zu rechnen, die durch Straftatbestände - abstrakten Gefährdungsdelikte - flankiert werden. Insofern ist das abstrakte Gefährdungsdelikt gegenwärtig der Prototyp des Wirtschaftsstrafrechts.

\section{Institutionenschutz und freiheitstheoretische Legitimation des abstrakten Gefährdungsdeliktes}

Damit sind wir bei der zweiten Frage angelangt: jene nach der Legitimation von abstrakten Gefährdungsdelikten. Diese lässt sich sowohl ökonomisch-krimino-

FAZ v. 31.7.2013, S. 19, Volk FS Schiller 2014, S. 672ff.; Wastl WM 2013, $1401 \mathrm{ff}$. Abweichend Kubiciel ZIS 2013, 53 ff.; ders. StV Editorial 10/2013.

28 Näher zu deren Schwächen Kubiciel (Fn. 25), S. 51 ff.

29 Anhand von Beispielen Kubiciel (Fn. 25), S. 2 f. 
logisch als auch juristisch-normativ behandeln und - abhängig vom methodischen Zugang - durchaus unterschiedlich beantworten. Eine empirischkriminologische Erörterung der Problematik muss beispielsweise prüfen, ob bzw. wie sicher sich prognostizieren lässt, dass ein bestimmtes (unternehmerisches) Verhalten fremdes Vermögen, eine einzelne Bank, das Kreditwesen o.ä. gefährdet. Man kann die Legitimitätsproblematik aber auch mit genuin strafrechtswissenschaftlichen Methoden bearbeiten, ohne dass in diesem Kontext die ökonomischen Erkenntnisse notwendig eine ausschlaggebende Bedeutung erlangen müssen. Denn der Nachweis tatsächlich vorhandener ökonomischer Risiken in einem Verhalten oder das Vorliegen eines tatsächlichen Schadens bzw. einer konkreten Gefahr ist nur von ausschlaggebender Bedeutung, wenn man dem Strafrecht allein oder jedenfalls vorrangig den Zweck zuweist, Rechtsgüter vor empirisch feststellbaren Gefährdungen oder Schäden zu schützen. Diese Konzeptualisierung des Strafrechtszwecks ist indes wissenschaftlich hoch umstritten auch deshalb, weil sie das Strafrecht nur unzureichend abbildet.

\section{Rechtsgüterschutz oder Normenschutz?}

Bereits im Jahr 1903 entdeckte Max Ernst Mayer das wirtschaftssteuernde Nebenstrafrecht: In der „entwickelten Rechtsordnung unserer Tage“ gebe es im Strafrecht „specialisierte Bestimmungen“, die nicht auf Kulturnormen beruhten, sondern nur für einen „besonderen Verkehr“ spezielle Pflichten statuierten. ${ }^{30}$ Die Zahl wirtschaftsverwaltungsrechtlicher Primärnormen, die unter Strafandrohung gestellt wurden, nahm während des 1 . Weltkrieges deutlich $\mathrm{zu}^{31}$ Eine Vielzahl von wirtschaftsstrafrechtlichen Gefährdungsdelikten fand Eingang in das fortan sogenannte Nebenstrafrecht. ${ }^{32}$ Bei all diesen Straftatbeständen steht der Rechtsgüterschutz nicht im Vorder- sondern im Hintergrund; primär sichern diese Straftatbestände die in Bezug genommenen außerstrafrechtlichen (Primär-)Normen. Diese Erkenntnis ist freilich älter als das Wirtschaftsstrafrecht. Gemeinhin wird sie Karl Binding zugeschrieben, ${ }^{33}$ doch verdankt sie sich keinem Geringerem als jenem Denker, der als Begründer der modernen und liberalen Strafrechtswissenschaft gilt: Paul Johann Anselm von

30 Mayer Rechtsnorm und Kulturnorm, 1903, S. 22ff.; zu Mayer Ziemann Jahrbuch der Jur. Zeitgeschichte 4 (2002/2003), $84 \mathrm{ff}$.

31 Dazu Tiedemann GA 1969, 71, $73 \mathrm{f}$.

32 Hirsch FS Tiedemann 2008, S. 145.

33 Grundlegend Binding Die Normen und ihre Übertretungen, Bd. 1, 4. Aufl. 1922. 
Feuerbach. ${ }^{34}$ Für Feuerbach war der Schutz subjektiver Rechte des Einzelnen und des Staates die Aufgabe des gesamten Strafrechts. ${ }^{35}$ Der Normschutzgedanke lässt sich folglich an liberale Traditionsbestände des strafrechtlichen Denkens anschließen.

Anders als von Feuerbach beabsichtigt schützen die abstrakten Gefährdungsdelikte jedoch nicht nur subjektive Rechte der Person, sondern - wie es heißt - hoch abstrakte, unbestimmte Gemeinschaftsrechtsgüter. ${ }^{36}$ Sind dies Anzeichen eines problematischen Sonderstrafrechts, ${ }^{37}$ das Züge der , historisch so diskreditierten Pflichtverletzungslehre“"38 trägt? Zunächst: Wer von einem Sonderstrafrecht spricht, muss das Normalstrafrecht begrifflich umreißen. Der Rechtsgüterschutzgedanke ist dazu, wie kein Geringerer als Hassemer ${ }^{39}$ betont hat, nicht mehr geeignet - wenn er diese Eignung denn je hatte. Denn der Rechtsgutsbegriff ist inhaltlich unbestimmt, ${ }^{40}$ sein Geltungsgrund unge-

34 Zur Berechtigung dieses Rufs Feuerbachs Kubiciel in: Koch/Kubiciel/Löhnig/Pawlik (Hrsg.), Feuerbachs Bayerisches Strafgesetzbuch, 2014, S.1, 11ff.; Frisch in: Koch/Kubiciel/ Löhnig/Pawlik (aaO), S. 191, $206 \mathrm{ff}$.

35 Näher dazu und zum Umgang Feuerbachs mit seiner Theorie Jakobs in: Koch/Kubiciel/ Löhnig/Pawlik (Fn. 34), S. $191 \mathrm{ff}$.

36 Weigend FS Frisch 2013, S. 17, 22. Krit. zur „eigentümlichen Unbestimmtheit“ der Rechtsgüter im Wirtschaftsstrafrecht zuletzt $H$. Schneider in: Brettel/Schneider, Wirtschaftsstrafrecht, 2014, §1 Rn. 84.

37 Prittwitz (Fn. 15), S. 350.

38 Davor warnend Kempf/Lüderssen/Volk in: dies. (Hrsg.), Gemeinwohl im Wirtschaftsstrafrecht, 2013, VII.

39 Hassemer zitiert bei Youssef/Godenzi, ZStW 125 (2013), 659, 665. - Anders Jahn in: Brodowski/Burchard (Hrsg.), Die Verfassung moderner Strafrechtspflege, im Erscheinen: „Für einen vollständigen Abschied von der Idee des Rechtsguts (...) besteht jedenfalls derzeit kein hinreichender Anlass. (...) Für die weitere Diskussion sollte ein Offensichtlichkeitsmaßstab herangezogen werden, der mir den nach wie vor berechtigten Kern der strafrechtlichen Rechtsgutslehre zutreffend abzubilden scheint. (...) Nur - aber auch gerade - dann, wenn eine Strafnorm für jeden Sachkundigen ohne längere Prüfung erkennbar keine Grundlage in einem mit der Verfassung kompatiblen Rechtsgut hat, ist es begründbar, dass sie gerade deshalb gegen Verfassungsrecht verstößt.“ Dem Verf., Matthias Jahn, sei für die freundliche Überlassung des Manuskripts herzlich gedankt.

40 S. nur Appel Verfassung und Strafe, 1998, S. 357 ff.; Frisch in: Hefendehl/v. Hirsch/Wohlers (Hrsg.), Die Rechtsgutstheorie: Legitimationsbasis des Strafrechts oder dogmatisches Glasperlenspiel?, 2003, S. 215, 217; Kuhlen in: Wolter/Freund (Hrsg.), Straftat, Strafzumessung im gesamten Strafrechtssystem, 1996, S. 77 (97); Wohlers Deliktstypen des Präventionsstrafrechts, 1993, S. 279; Stuckenberg GA 2011, 653, 657. - Klassisch die Kritik v. Liszts Strafrechtliche Vorträge und Aufsätze, Bd. 1, 1970, S. 224, aus dem Jahr 1873: Der Rechtsgutsbegriff (namentlich Bindings) sei ein „Proteus, der alle Gestalten annimmt; ein Wort, das heute das und 
klärt, ${ }^{41}$ und selbst Vertreter einer systemkritischen Rechtsgutskonzeption setzen sich über deren Grenzen hinweg, wenn es ihnen kriminalpolitisch opportun erscheint. ${ }^{42}$ Auch mit Hilfe eines Verweises auf eine bessere Vergangenheit lässt sich nach meinem Dafürhalten ein legitimes „Normalstrafrecht“ nicht (allein) bestimmen. Anders gewendet: Dass vor 40 Jahren noch das Erfolgsdelikt der Prototyp des (Wirtschafts-)Strafrecht im StGB war, ist kein Argument gegen das abstrakte Gefährdungsdelikt als neuen Prototyp. Denn ebenso wenig wie Liszt von einem gegenwärtigen Recht auf das künftig sein-sollende Recht schließen kann, lässt sich das heutige Recht am Maßstab des vergangenen Rechts prüfen. Beide Sichtweisen leben von unausgesprochenen geschichtsmetaphysischen Annahmen, die weit von allgemeiner Anerkennung entfernt sind, um das Wenigste $\mathrm{zu}$ sagen. Unabhängig davon wäre zu begründen, an welcher historischen Stunde das heutige Strafrecht Maß nehmen müsste. Die legitimen Grenzen des Strafrechts lassen sich nicht an der Gestalt des Strafgesetzbuchs von 1871 oder 1975 ablesen; maßgeblich muss das Jahr 2014 sein. Anders gewendet: Das Strafrecht lässt sich nicht auf einen - wie auch immer zu bestimmenden historischen Status quo „einfrieren“, sondern muss sich den Veränderungen der gesellschaftlichen Struktur anpassen. ${ }^{43}$ Es muss, nochmals anders gewendet, auf der Höhe seiner Zeit sein, ${ }^{44}$ in der es seinen Beitrag zur Aufrechterhaltung eines Zustandes von Freiheit leisten soll.

\section{Institutionenschutz als Freiheitsschutz}

Freiheit, wie wir sie heute verstehen, ist aber nicht lediglich die Abwesenheit von Zwang; Freiheit ist die reale Möglichkeit zur personalen Selbstentfaltung. ${ }^{45}$

morgen wieder etwas ganz anderes bedeutet, ein Blankett, dem jeder den Inhalt geben kann, der ihm gerade passt.“

41 Dazu zuletzt Hilgendorf NK 2010, 125, 128 f.; Stuckenberg GA 2011, 653 ff.

$42 \mathrm{Zu}$ „Erweiterungen des strafrechtlichen Regelungsbereichs über den Rechtsgüterschutz hinaus“ s. Roxin Strafrecht AT I, 4. Aufl. 2006, § 2 Rn. 29 ff.

43 Hirsch FS Tiedemann 2008, S. 145, 155.

44 Jakobs in: Engel/Schön (Hrsg.), Das Proprium der Rechtswissenschaft, 2007, S. 134, 136. Weiterführend Pawlik GA 2014, 301ff.; ders. FS Heintschel-Heinegg 2015. - In diesem Sinne ist das Strafrecht stets politisch, vgl. Prittwitz Fachbereich Rechtswissenschaft der GoetheUniversität Frankfurt am Main, 100 Jahre Rechtswissenschaft in Frankfurt, 2014, S. 343, 345, der zutreffend meint, man könne Strafrecht gar nicht unpolitisch betreiben.

45 Näher Kubiciel in: Koch/Kubiciel/Löhnig/Pawlik (aaO), S. 393, 408ff.; Pawlik Das Unrecht des Bürgers, 2012, S. $90 \mathrm{ff}$. 
Für die personale Selbstentfaltung sind Institutionen von erheblicher Bedeutung. Institutionen lassen sich definieren als rechtlich garantierte Bedingungen freier Entfaltung in einer Gesellschaft. ${ }^{46}$ Das Eigentum beispielsweise ist eine solche Einrichtung, die intersubjektive Freiheitsräume definiert und die Möglichkeit personaler Entfaltung vorstrukturiert. ${ }^{47}$ Als Institution ist das Eigentum den Einzelnen vorgegeben. Gerade weil eine Institution nicht Gegenstand permanenter intersubjektiver Aushandlungen ist, kann sie einen bereichsspezifischen Sinn und erprobte Vernünftigkeits- und Gerechtigkeitsstandards sowie daraus abgeleitete Regeln speichern. ${ }^{48}$ Das Vorhandensein einer auf Dauer gestellten Institution, deren Regeln sich über die Zeit und in der sozialen Praxis bewährt haben, bietet Orientierung und ermöglicht es dem Einzelnen, sich in persönlicher, wirtschaftlicher und unternehmerischer Hinsicht frei zu entfalten. Auch der Wettbewerb und das Kreditwesen sind schutzwürdige Institutionen, ${ }^{49}$ die durch Normen nach außen verfestigt und nach innen ausgestaltet werden. ${ }^{50}$

Institutionen als Ideen von einer sozialen Praxis müssen nicht nur tatsächlich in der Gesellschaft verankert sein, sondern müssen - damit sie ihre Funktionen auf Dauer ausüben können - auch rechtlich geschützt werden. ${ }^{51}$ Normen, die für die Stabilität und die Funktion einer Institution unabdingbar sind, bedürfen daher eines (strafrechtlichen) Schutzes. ${ }^{52}$ So gesehen, ist es zu pauschal, dem (Wirtschafts-)Strafrecht und seinen abstrakten Gefährdungsdelikten vorzuwerfen, es schütze nicht nur Individualrechtsgüter, sondern diffuse Kollektivrechtsgüter. Abstrakte Gefährdungsdelikte wie §264a StGB, aber auch §54a KWG garantieren Regeln, die für den Bestand und die Funktion der jeweiligen Institution von Bedeutung sind. Die Garantie dieser normativen Funktionsbe-

46 Jakobs Rechtsgüterschutz? Zur Legitimation des Strafrechts, 2012, S. 26. S. ferner Lampe FS Tiedemann 2008, S. 79, 83; Kindhäuser Zur Legitimität der abstrakten Gefährdungsdelikte im Wirtschaftsstrafrecht, in: Schünemann/Suárez Gonzáles (Hrsg.), Bausteine eines europäischen Wirtschaftsstrafrechts, 1994, S. 125, 128.

47 Vgl. Zabel in: Seelmann/Zabel (Hrsg.), Autonomie und Normativität. Zu Hegels Rechtsphilosophie, 2014, S. 153, 158f., im Anschluss an Nussbaum Die Grenzen der Gerechtigkeit, 2010, S. 420.

48 Ähnlich Zabel in: Seelmann/Zabel (Fn. 47), S. 165.

49 Anders Kindhäuser (Fn. 46), S. 129: Das Kreditwesen gehöre nicht zu den rechtlich garantierten Bedingungen freier Entfaltung.

50 Zur Notwendigkeit, Institutionen durch Normen ,juristisch zu verfestigen“, vgl. Lampe (Fn. 46), S. 97.

51 Vgl. zu der von ihm so genannten „Sachinstitution“ Hauriou Die Theorie der Institution, hrsg. von Roman Schnur1965, S. 35.

52 Kindhäuser FS Krey 2010, S. 249, 265. Weitergehende Voraussetzungen bei Schünemann GA 1995, 201, $213 \mathrm{f}$. 
dingungen von Institutionen ist auch freiheitstheoretisch notwendig, weil eine Institution, deren Bestand oder Funktionsweise unsicher ist, gerade nicht zur Gewährleistung von Vertrauen und personaler Freiheit geeignet ist. ${ }^{53}$ In die Sprache des Strafverfassungsrechts gewendet bedeutet das: Der Gesetzgeber bewegt sich im „weiten Raum verfassungsrechtlicher Freiheit“, in dem er Verhaltensnormen mit strafrechtlichen Sanktionsnormen bewehren darf, ${ }^{54}$ wenn er abstrakte Gefährdungsdelikte einsetzt, um Normen zu sichern, die für den Bestand einer freiheitsermöglichenden Institution wichtig sind. Da diese strafbewehrten Normen nur jene erfassen, die sich innerhalb dieser Institutionen oder „konkreten Erfahrungsräumen“ bewegen (und von ihnen profitieren), kann auch von einer übermäßigen Freiheitsbeschränkung keine Rede sein. ${ }^{55}$

\section{Ergebnisse}

1. Auch wenn simplifizierenden Evolutionstheorien des Rechts und daraus abgeleiteten Prognosen mit Skepsis zu begegnen ist (II. 1.), gibt es Indizien, die für einen Trend zum abstrakten Gefährdungsdelikt als Prototyp des Wirtschaftsstrafrechts sprechen (II. 3.).

2. Abstrakte Gefährdungsdelikte schützen Normen, keine Güter (III. 1.). Dass eine konkrete Tat den Wettbewerb, das Kreditwesen oder dergleichen nicht empirisch nachweisbar schädigen oder gefährden kann, ${ }^{56}$ ist für die Legitimation der Straftatbestände daher nicht von Bedeutung.

3. Legitim sind solche Straftatbestände insbesondere dann, wenn sie die Geltung solcher Normen garantieren, die für die Stabilität einer freiheitsermöglichenden Institution notwendig sind (III.2.).

4. Dies bedeutet, dass die Deliktstypenlehre kein Seismograph für die Liberalität oder Illiberalität des Strafrechts ist. Konkreter gesprochen: An der Anzahl von abstrakten Gefährdungsdelikten im Strafrecht lässt sich nicht ablesen, ob das Strafrecht freiheitsfreundlich oder freiheitsfeindlich ist. Die Frage der

53 Vgl. Kindhäuser (Fn. 46), S. 131: Ein Rechtsgut, über das nicht unbesorgt verfügt werden kann, sei nicht in vollem Umfange nutzbar und daher geschädigt.

54 Vgl. Jahn (Fn. 39). Zum weiten verfassungsrechtlichen Rahmen, innerhalb dessen der Gesetzgeber seinen kriminalpolitischen Beurteilungsspielraum nutzen kann, auch Gärditz Der Staat 49 (2010), 331, 341ff.; Stuckenberg GA 2011, 653ff. Am Beispiel des Unternehmensstrafrechts Kubiciel ZRP 2014, $133 \mathrm{ff}$.

55 Ähnlich Mansdörfer Zur Theorie des Wirtschaftsstrafrechts, 2011, S. 83f.

56 Kindhäuser (Fn. 46), S. 129. 
Strafwürdigkeit und jene nach dem Deliktstyp sind auf unterschiedlichen Ebenen angesiedelt. ${ }^{57}$ Der Prototyp „abstraktes Gefährdungsdelikt diskreditiert daher auch das Wirtschaftsstrafrecht nicht. Im Gegenteil: Auch und gerade ein liberal-freiheitssicherndes Strafrecht kommt ohne diese Reaktionsform auf Wirtschaftskriminalität nicht aus. Denn wenn das Strafrecht nicht auf Verhaltensweisen reagiert, die verbreitet für strafwürdig erachtet werden, steht nicht nur die Geltung einer einzelnen Norm oder einer Institution auf dem Spiel. Es geht, in letzter Konsequenz, um nicht weniger als die Akzeptanz der sozialen Marktwirtschaft. ${ }^{58}$

\section{Cornelius Prittwitz}

Vielen Dank, Herr Kubiciel. Ich bin ganz sicher, dass ich nicht der einzige bin, der sofort am liebsten in eine engagierte und unter Umständen auch kontroverse Diskussion eintreten würde, aber Sie haben sich, was ich ganz wunderbar und anregend finde, in die Höhle des Frankfurter Löwen begeben, in der die abstrakten Gefährdungsdelikte überwiegend anders betrachtet werden. Nicht ganz einhellig, wir kommen darauf noch zu sprechen. Zuerst hören wir, was der Ökonom zu alledem zu sagen hat: Herr Wahrenburg, bitte.

57 Kindhäuser (Fn. 52), S. 249, 264.

58 Richter in: Müller-Gugenberger/Bieneck (Hrsg.), Wirtschaftsstrafrecht, 5. Aufl. 2011, § 2 Rn. 50. 